\title{
Weight-reduction through a low-fat diet causes differential expression of circulating microRNAs in obese C57BL/6 mice
}

\author{
Ching-Hua Hsieh ${ }^{1 * \dagger}$, Cheng-Shyuan Rau ${ }^{2 \dagger}$, Shao-Chun $\mathrm{Wu}^{3+}$, Johnson Chia-Shen Yang ${ }^{1}$, Yi-Chan Wu',
} Tsu-Hsiang Lu', Siou-Ling Tzeng ${ }^{1}$, Chia-Jung Wu ${ }^{1}$ and Chia-Wei Lin ${ }^{1}$

\begin{abstract}
Background: To examine the circulating microRNA (miRNA) expression profile in a mouse model of diet-induced obesity (DIO) with subsequent weight reduction achieved via low-fat diet (LFD) feeding.

Results: Eighteen C57BL/6NCrl male mice were divided into three subgroups: (1) control, mice were fed a standard AIN-76A (fat: $11.5 \mathrm{kcal} \%$ ) diet for 12 weeks; (2) DIO, mice were fed a $58 \mathrm{kcal} \%$ high-fat diet (HFD) for 12 weeks; and (3) DIO + LFD, mice were fed a HFD for 8 weeks to induce obesity and then switched to a $10.5 \mathrm{kcal} \%$ LFD for 4 weeks. A switch to LFD feeding led to decreases in body weight, adiposity, and blood glucose levels in DIO mice. Microarray analysis of miRNA using The Mouse \& Rat miRNA OneArray ${ }^{\circledR}$ v4 system revealed significant alterations in the expression of miRNAs in DIO and DIO + LFD mice. Notably, 23 circulating miRNAs (mmu-miR-16, mmu-let-7i, mmu-miR-26a, mmu-miR-17, mmu-miR-107, mmu-miR-195, mmu-miR-20a, mmu-miR-25, mmu-miR-15b, mmu-miR-15a, mmu-let-7b, mmu-let-7a, mmu-let-7c, mmu-miR-103, mmu-let-7f, mmu-miR-106a, mmu-miR-106b, mmu-miR-93, mmu-miR-23b, mmu-miR-21, mmu-miR-30b, mmu-miR-221, and mmu-miR-19b) were significantly downregulated in DIO mice but upregulated in DIO + LFD mice. Target prediction and function annotation of associated genes revealed that these genes were predominantly involved in metabolic, insulin signaling, and adipocytokine signaling pathways that directly link the pathophysiological changes associated with obesity and weight reduction.
\end{abstract}

Conclusions: These results imply that obesity-related reductions in the expression of circulating miRNAs could be reversed through changes in metabolism associated with weight reduction achieved through LFD feeding.

Keywords: Diet-induced Obesity, MicroRNAs, High-fat Diet, Low-fat Diet

\section{Background}

Obesity is associated with insulin resistance and an abnormal inflammatory response [1], and the strong associations suggest that adipose tissue plays a prominent role in the onset and progression of these comorbidities [2]. White adipose tissue (WAT) has been characterized as an endocrine organ [3], as it produces endocrineacting peptides such as leptin, and it is metabolically important, with excess levels being associated with metabolic syndrome $[4,5]$. High fat uptake leads to

\footnotetext{
* Correspondence: m93chinghua@gmail.com

${ }^{\dagger}$ Equal contributors

'Department of Plastic and Reconstructive Surgery, Kaohsiung Chang Gung Memorial Hospital and Chang Gung University College of Medicine, No. 123, Ta-Pei Road, Niao-Song District, Kaohsiung City 833, Taiwan

Full list of author information is available at the end of the article
}

metabolic alterations in adipose tissue that increase the levels of circulating free fatty acids in the blood [6]. This leads to macrophage activation and the production of proinflammatory cytokines via Toll-like receptors, resulting in inflammation in adipose tissue [6]. When allowed ad libitum access to a high-fat diet (HFD), C57BL/6J mice develop insulin resistance and obesity in a manner that resembles disease progression in humans [7]. Increased energy expenditure and decreased energy intake are the two most commonly recommended lifestyle changes to reduce adiposity and restore insulin sensitivity in the treatment of diet-induced obesity (DIO) and associated comorbidities [8]. Calorie restriction is effective in improving insulin sensitivity and decreasing both body weight and percent body fat [9]. In addition, reductions in

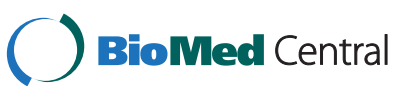

(C) 2015 Hsieh et al. Open Access This article is distributed under the terms of the Creative Commons Attribution 4.0 International License (http://creativecommons.org/licenses/by/4.0/), which permits unrestricted use, distribution, and reproduction in any medium, provided you give appropriate credit to the original author(s) and the source, provide a link to the Creative Commons license, and indicate if changes were made. The Creative Commons Public Domain Dedication waiver (http://creativecommons.org/publicdomain/zero/1.0/) applies to the data made available in this article, unless otherwise stated. 
body weight and improvements in insulin sensitivity can also be achieved by reducing the percentage fat in a diet, i.e., by switching from a HFD to a low-fat diet (LFD) [10].

MicroRNAs (miRNAs) are endogenous small RNAs that post-transcriptionally regulate gene expression, and they have been demonstrated to have important roles in numerous disease processes. There is growing evidence that miRNAs play an important role in regulating adipose tissue pathways that control a range of processes, including adipogenesis, insulin resistance, and inflammation [11-13]. Many miRNAs are dysregulated in the metabolic tissues of obese animals and humans, potentially contributing to the pathogenesis of obesity-associated complications [11-13]. In addition, recent studies identified several miRNAs expressed in metabolic organs that could be used as feasible therapeutic targets for obesity and its consequent pathologies $[11,13]$. Recently, circulating serum miRNAs were found to display specific expression patterns, suggesting that miRNA profiles may represent fingerprints for various diseases $[14,15]$. In addition, despite the ubiquitous presence of ribonucleases, serum miRNAs levels are remarkably stable and reproducible [16, 17], and they function in cell-to-cell communication [18]. Currently, how changes in miRNA profiles might affect adipose tissue at the functional and molecular level and to what extent they differ in response to weight-reduction strategies are not well understood. This information is important in the development of dietary anti-obesity interventions [19]. As circulating miRNAs potentially play an important role in regulating the pathophysiology of obesity and they are potential therapeutic targets, we hypothesized the weight reduction may change the circulating miRNAs expression. Our study aim was to profile the expression of circulating miRNAs in a mouse model of DIO with subsequent weight reduction achieved through LFD feeding.

\section{Methods}

\section{Ethics statement}

This study was conducted in strict accordance with guidelines on the use of laboratory animals, and every effort was made to minimize the suffering of affected animals. Animal protocols were approved by the IACUC of Chang Gung Memorial Hospital, Taiwan (permission number No. 2012091002).

\section{Animal experiments}

C57BL/6NCrl mice were purchased from BioLasco (Taipei, Taiwan). Animals were housed, and surgical procedures, including analgesia, were performed in an Association for Assessment and Accreditation of Laboratory Animal Care International-accredited SPF facility according to national and institutional guidelines. In this experiment, 18 male, wild-type $\mathrm{C} 57 \mathrm{BL} / 6 \mathrm{NCrl}$ mice were randomly assigned to three subgroups ( $n=6$ in each group) as follows: (1) control, mice were fed a standard AIN-76A (fat: 11.5 kcal \%) diet ad libitum for 12 weeks; (2) DIO, mice were fed a $58 \mathrm{kcal} \%$ HFD (D12331; Research Diets Inc., New Brunswick, NJ) ad libitum for 12 weeks to induce obesity; and (3) DIO + LFD, mice were fed a $58 \mathrm{kcal} \%$ HFD (D12331) ad libitum for 8 weeks to induce obesity and then fed a $10.5 \mathrm{kcal} \%$ LFD (D 12329; Research Diets Inc.) for 4 weeks. Weight measurements were performed weekly, and a glucose tolerance test was performed at the beginning and end of the experiment to confirm that HFD-fed mice developed an obese and glucose intolerance phenotype. Briefly, mice were fasted for $5 \mathrm{~h}$, and baseline blood glucose levels were measured with an Accu-Check Advantage blood glucose meter (Roche, New Jersey, USA) using blood collected from the tail vein. Mice ( $n=6$ in each group) were injected intraperitoneally with $2 \mathrm{~g}$ of glucose per kilogram body weight in sterile PBS. The glucose level was measured via tail vein blood $(\sim 10 \mu \mathrm{L})$ at $\mathrm{t}=-30$ and 0 (pre) and $\mathrm{t}=15$, $30,60,90$, and $120 \mathrm{~min}$ after the glucose infusion. Data were averaged and graphed as blood glucose level as a function of time. To reflect the circulating levels of glucose during the glucose tolerance test (GTT), we calculated the total area under the curve (AUC) of the glucose concentration versus time by the linear trapezoidal rule for the period of $0-120$ min after glucose infusion. To avoid the effect of loss of blood in GTT experiment or uncertain effect or repeat tail punctures on subsequent miRNAs expression and cytokine assay, additional groups of mice under the same model were used for further experiments. After the end of the experiment, all mice were euthanized, and the abdominal mesenteric WAT of each mouse was removed and weighed. The adipose tissue block embedded in paraffin was sectioned at $5 \mu \mathrm{m}$ to measure the adipocyte area. Three $5 \mu \mathrm{m}$-thickness sections of the same fat specimen at $50 \mu \mathrm{m}$ distance was mounted on glass plate and stained with hematoxylin and eosin. Two different microscopic fields (magnification $\times 100)$ per plate were photographed and 100 adipose cells were arbitrarily selected in the center of field and their cell diameters were assessed by tracing the outline of each adipocyte. The mean adipocyte area was measured from the WAT of control and experimental mice ( $n=4$ in each group) using Image-Pro Plus image analysis software (Carl Zeiss, Oberkochen, Germany) and expressed in terms of square micrometers. The cells were randomly chosen, and the person analyzing the images was blinded to the group assignments. At the indicated time of the experiment, $1 \mathrm{~mL}$ of whole blood was collected via cardiac puncture into a plain tube and 
allowed to clot for $1 \mathrm{~h}$. Samples were centrifuged at $3000 \times g$ for $10 \mathrm{~min}$, and sera were aliquoted and stored at $-80^{\circ} \mathrm{C}$ until further analysis.

\section{Cytokine assays}

Serum cytokine concentrations were analyzed using two complementary Bio-Plex suspension arrays (M6000003J7 and M60-00007NY) covering all cytokine biomarkers potentially involved in inflammation $(n=6$ in each group). Eleven biomarkers (IL-1 $\beta$, IL-2, IL-4, IL5, IL-6, IL-10, IL-12(p70), IL-17, GM-CSF, IFN- $\gamma$, and TNF- $\alpha$ ) were assessed simultaneously using the BioPlex system (BioRad, Hercules, CA). Assays were performed on four biological replicates per the manufacturer's instructions. Results are expressed in picograms per milliliter of serum.

\section{RNA isolation and preparation}

Total RNA was extracted from serum using the mirVana $^{\text {Tw }}$ miRNA Isolation Kit (Life Technologies, NY). Purified RNA was quantitatively evaluated by measuring its absorbance at $260 \mathrm{~nm}$ using an SSP-3000 Nanodrop spectrophotometer (Infinigen Biotechnology, Inc., City of Industry, CA), and RNA quality was assessed using a Bioanalyzer 2100 (Agilent Technologies, Santa Clara, CA). Total RNA (10 ng) was reverse-transcribed into cDNA using a TaqMan miRNA Reverse Transcription Kit (Applied Biosystems, Foster City, CA). Target miRNAs were reverse-transcribed using sequence-specific stem-loop primers, and cDNA was used for quantitative real-time polymerase chain reaction (qPCR).

\section{miRNA microarray analysis}

The Mouse \& Rat miRNA OneArray v4 (Phalanx Biotech Group, Hsinchu, Taiwan) array used in this experiment contains 144 experimental control probes, 1157 unique mouse miRNA probes, and 680 rat miRNA probes, based on the miRBase 18 database. Three biological replicates of each group of mice were used in miRNA microarray experiments. Mouse genome-wide miRNA microarray experimental and statistical analyses were performed by Phalanx Biotech Group. Briefly, fluorescent targets were prepared from $2.5 \mu \mathrm{g}$ of total RNA using the miRNA ULS ${ }^{\text {Tx }}$ Labeling Kit (Kreatech Diagnostics, Amsterdam, Netherlands). Labeled miRNA targets enriched using NanoSep 100K (Pall Corporation, Port Washington, NY) were hybridized to The Mouse \& Rat miRNA OneArray ${ }^{\circ} 4$ in Phalanx hybridization buffer in the OneArray Hybridization Chamber. After overnight hybridization at $37{ }^{\circ} \mathrm{C}$, non-specifically bound targets were removed by three washing steps (Wash I, $37{ }^{\circ} \mathrm{C}, 5 \mathrm{~min}$; Wash II, $37{ }^{\circ} \mathrm{C}$, 5 min and $25{ }^{\circ} \mathrm{C}, 5 \mathrm{~min}$; and Wash III, rinse 20 times). Slides were dried by centrifugation and scanned using an
Axon 4000B scanner (Molecular Devices, Sunnyvale, CA). The signal intensities of Cy5 fluorescence in each spot were analyzed using GenePix 4.1 software (Molecular Devices, Sunnyvale, CA) and processed using R language (http://www.r-project.org/) with two packages: limma (http://www.bioconductor.org/packages/release/bioc/html/ limma.html) and genefilter (http://www.bioconductor.org/ packages/release/bioc/html/genefilter.html). Spots with flag $<0$ were filtered out, and the remaining spots were $\log$ 2 transformed and normalized using the $75 \%$ media scaling normalization method. Normalized spot intensities were converted into gene expression ratios between the control and treatment groups. Spots with expression ratios $\leq 0.5$ or $\geq 2$, as well as with $p<0.05$, were selected for further analysis. Differentially expressed miRNAs were subjected to hierarchical cluster analysis using average linkage and Pearson's correlation as the measure of similarity. The miRNA array data have been deposited in the NCBI Gene Expression Omnibus with the accession number GSE61005. Five miRNAs detected by array analysis were selected and quantified by qPCR using the Applied Biosystems 7500 Real-Time PCR System (Life Technologies) to confirm the upregulation of miRNA expression in the DIO + LFD group. Twenty-five femtomoles of single-stranded cel-miR-39 synthesized by Invitrogen (Carlsbad, CA) was spiked into $400 \mu \mathrm{L}$ of serum as an internal control for the expression of each miRNA.

\section{Target prediction, GO enrichment, and KEGG pathway analyses}

Target prediction was performed to identify the target genes of the identified dysregulated miRNAs by integrating all three public databases (TargetScan, PicTar, and miRanda). This method firstly mapped all target gene candidates to GO terms in the database (http:// www.geneontology.org/), calculated gene numbers for each term, and then used a hypergeometric test to find significantly enriched GO terms in target gene candidates compared to the reference gene background. Bonferroni's correction for the $p$-value was used to obtain a corrected $p$-value. $\mathrm{GO}$ terms with corrected $p$-values $\leq 0.05$ were defined as significantly enriched in target gene candidates. To reveal the main pathways in which the target gene candidates are involved, pathway analysis using a major public pathway-related database, KEGG, was performed to identify significantly enriched metabolic pathways or signal transduction pathways in target gene candidates compared with the whole reference gene background. Genes with $\mathrm{FDR} \leq 0.05$ were considered significantly enriched among the target gene candidates.

\section{Statistical analysis}

The body weight of mice are expressed as the mean with a confidence interval. All other experimental data are 


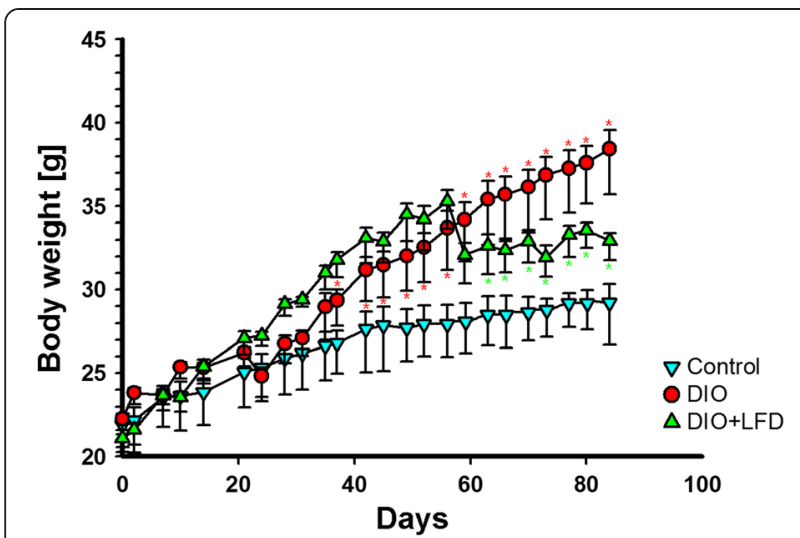

Fig. 1 The body weight of C57BL/6NCrl mice on a weekly basis till 12 weeks ( $n=6$ in each group). Control: the mice fed with the standard diet; DIO: the mice fed with high-fat diet; DIO + LFD: the mice fed with high-fat diet for 8 weeks to induce obesity, then change to low-fat diet for subsequent 4 weeks. *(red color), $P<0.05$ vs. control. * (green color), $P<0.05$ vs. DIO. The body weight of mice are expressed as the mean a $\pm 80 \%$ confidence interval

expressed as the mean \pm standard error of the mean. Analysis of variance combined with a Bonferroni post hoc correction was performed to identify significant differences in body weight, weight of fat, adipocyte area, glucose levels, and serum cytokine levels. A $p$ value of 0.05 was regarded as the level of statistical significance.

\section{Results}

\section{LFD decreased body weight and adiposity}

In comparison with the $\mathrm{C} 57 \mathrm{BL} / 6 \mathrm{NCrl}$ mice fed the standard diet, feeding with the HFD significantly increased body weight (Fig. 1). By contrast, after the change from HFD feeding to LFD feeding, the body weight of DIO mice decreased quickly, with the mean body weight stabilizing after 4 weeks on the LFD. Abdominal mesenteric WAT was also significantly larger in the DIO mice than in control mice, and the mice that were switched to the LFD had significantly less abdominal mesenteric WAT than those that remained on the HFD (Fig. 2a). However, the lower amount of abdominal mesenteric WAT was not accompanied by a significantly smaller adipocyte area (Figs. 2b and 3). Notably, because the fat specimen chosen at 50 $\mu \mathrm{m}$ distance was less than the mean diameter of the adipocytes, there may be exist a selection bias that some measured adipocytes were repeatedly calculated. However, we expect this selection bias is not significant because the tissue section was chosen in a fixed distance and the counting of high number of adipocytes could decrease the deviation of calculated mean adipocyte area. After injection of glucose to the control mice, blood glucose levels increased to a peak of $350 \mathrm{mg} / \mathrm{dL}$ after $15 \mathrm{~min}$, and then gradually returned to baseline after $120 \mathrm{~min}$. In the DIO mice, blood glucose concentrations at 30 to 120 min during the GTT were significantly higher than those in the control mice (Fig. 4a). HFD-fed animals displayed significant impairment in glucose tolerance, as evidenced by a $90 \%$ higher incremental glucose AUC (Fig. 4b). In addition, significantly lower glucose level was observed at 30 min after glucose injection for the LFD-fed mice relative to the HFD-fed mice (Fig. 4a), resulting in an around $15 \%$ lower glucose AUC (Fig. 4b).

\section{LFD did not induce the differential expression of inflammatory cytokines}

Of the 11 biomarkers measured (IL-1 $\beta$, IL-2, IL-4, IL-5, IL-6, IL-10, IL-12 (p70), IL-17, GM-CSF, IFN- $\gamma$, and
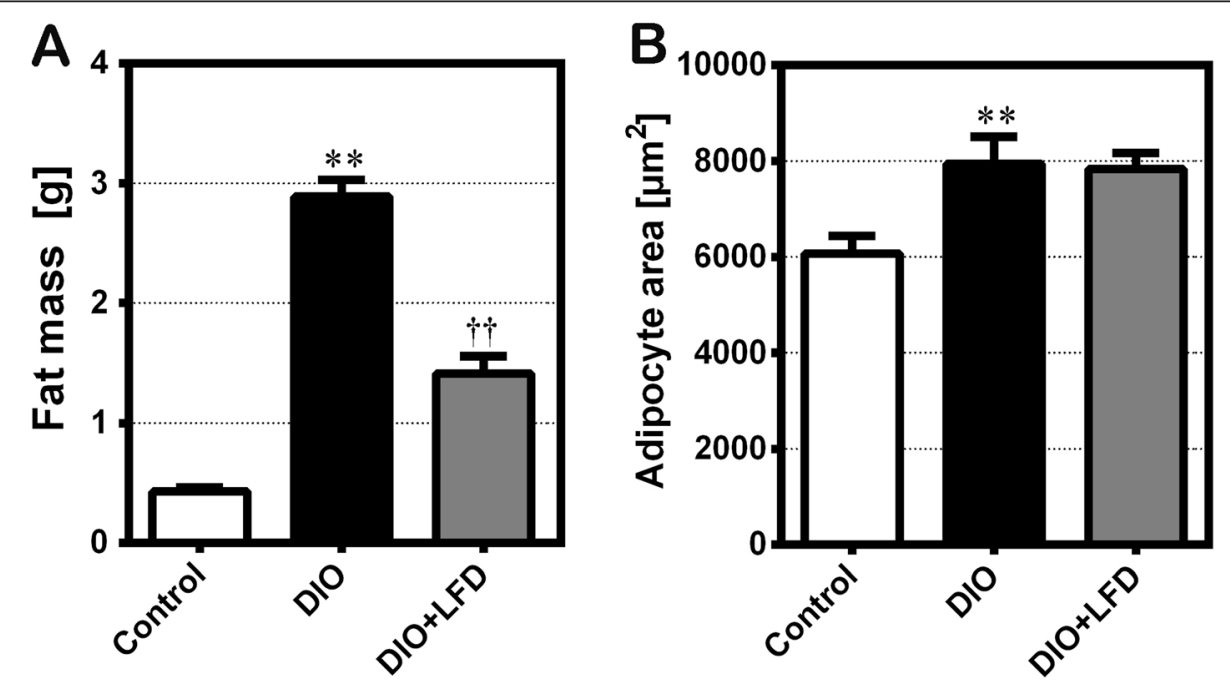

Fig. 2 a The weight of abdominal mesenteric white adipose tissue and $\mathbf{b}$ the adipocyte area of C57BL/6NCrl mice in groups of control, DIO, DIO + LFD at $12 \mathrm{w} .{ }^{* *}, P<0.01$ vs. control. + 十, $P<0.01 \mathrm{vs}$. DIO ( $n=4$ in each group) 




Fig. 3 Hematoxylin and eosin stain of the paraffin-embedded white adipose tissue at $5 \mu \mathrm{m}$ section of C57BL/6NCrl mice in groups of control, $\mathrm{DIO}, \mathrm{DIO}+\mathrm{LFD}$ at $12 \mathrm{w}$

TNF- $\alpha$ ), IL-1 $\beta$ had the highest expression in serum, followed by TNF- $\alpha$, IL-10, and IL-12 (p70) (Fig. 5). However, there were no significant differences in expression among the groups.

\section{Upregulated miRNA targets in microarray analysis}

Greater than 2-fold differences in serum miRNA expression between DIO mice and controls as well as between DIO + LFD and DIO mice $(p<0.05)$ were identified for further analysis. The microarray and qPCR results were in agreement, with a Pearson correlation value of 0.891 (Additional file 1), under the limitation of only five miRNAs being selected for analysis. Unsupervised hierarchical clustering of all differentially expressed serum miRNAs was conducted to separate samples from experimental or control subjects into different groups (Fig. 6). In microarray experiments of DIO mouse sera, eight miRNAs were upregulated, and 34 were downregulated (Table 1 ). In addition, in the sera of $\mathrm{DIO}+$ LFD mice, 28 miRNAs were upregulated, and 10 were downregulated (Table 2). As shown in the Venn diagram in Fig. 7, notably, 23 of the 28 upregulated miRNAs in DIO + LFD mice (mmu-miR-16, mmu-let-7i, mmu-miR-26a, mmu-miR-17, mmu-miR-107, mmu-miR-
195, mmu-miR-20a, mmu-miR-25, mmu-miR-15b, mmumiR-15a, mmu-let-7b, mmu-let-7a, mmu-let-7c, mmumiR-103, mmu-let-7f, mmu-miR-106a, mmu-miR-106b, mmu-miR-93, mmu-miR-23b, mmu-miR-21, mmu-miR30b, mmu-miR-221, and mmu-miR-19b) were downregulated in the DIO mice. Only five miRNAs (mmu-miR451, mmu-miR-223, mmu-miR-92a, mmu-miR-200c, and mmu-miR-873) were differentially expressed, implying that the majority of miRNA downregulation associated with obesity could be reversed by LFD treatment. By contrast, of the eight upregulated miRNAs in DIO mice, only one (mmu-miR-711) was significantly downregulated in DIO + LFD mice.

\section{Target prediction and function annotation}

To further understand the physiological functions and biological processes associated with the 23 miRNAs, target prediction was performed by integrating three public databases (TargetScan, PicTar, and miRanda), and 1082 target genes were identified. GO annotation and KEGG pathway analysis was also performed to identify functional modules regulated by these $23 \mathrm{miR}$ NAs. In GO annotation analysis, cellular processes, biological regulation, metabolic processes, primary


Fig. 4 Blood glucose concentrations (a) and area under the curve (AUC) quantification (b) during a 120-min glucose tolerance test in groups of control, DIO, DIO + LFD at $12 \mathrm{w}$. ${ }^{*}$ (red color), $P<0.05$ vs. control. * (green color), $P<0.05$ vs. DIO 


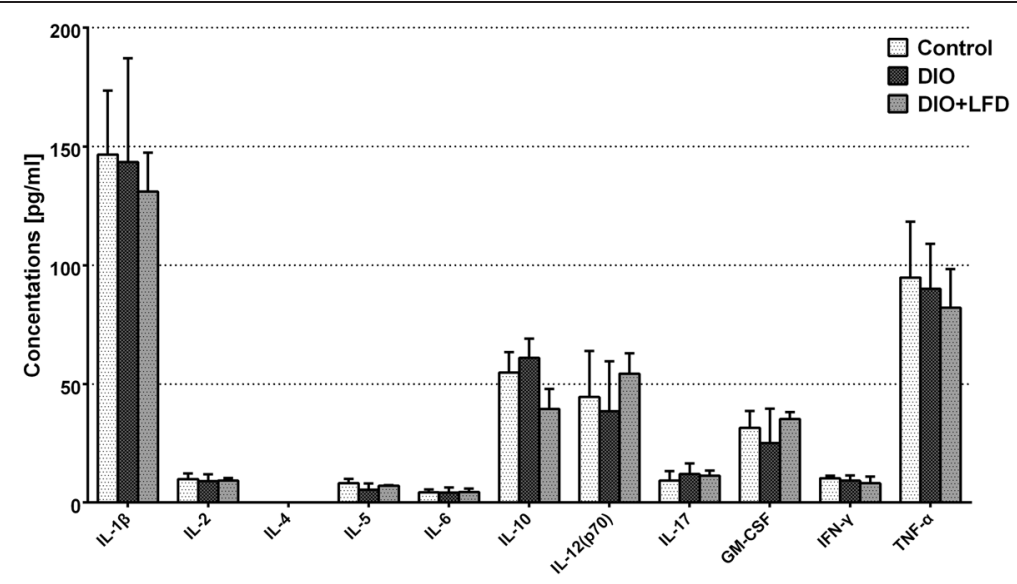

Fig. 5 Concentrations of serum cytokines (IL-1 $\beta$, IL-2, IL-4, IL-5, IL-6, IL-10, IL-12(p70), IL-17, GM-CSF, IFN- $\gamma$, TNF-a) analyzed by the Bio-Plex Multiplex cytokine assay at $12 \mathrm{w}$ in the mice in groups of control, DIO, DIO + LFD ( $n=6$ in each group)

metabolic processes, and cellular metabolic processes were the most significantly enriched GO terms (Fig. 8). KEGG pathway analysis revealed 142 pathways associated with these miRNA targets. Among these, metabolic pathways were the most enriched, with 1024 associated genes, followed by MAPK signaling, actin cytoskeleton regulation, secondary metabolite biosynthesis, focal adhesion, insulin signaling, calcium signaling, cytokine-cytokine receptor interaction, tight junctions, phagosomes, and adipocytokine signaling pathways (Table 3). These results suggest that these targets have a high possibility of being regulated by miRNAs during obesity and weight reduction through LFD feeding; however, the possibility of false-positive results from the prediction algorithm always exists.

\section{Discussion}

Switching to a LFD is an effective intervention to promote weight loss and improve metabolic health parameters in obesity [19]. Although morbid obesity is considered a systemic inflammatory state, the serum inflammatory profile of C57BL/6 mice, as measured by an antibody array, revealed that DIO mice had higher leptin, IL-6, and LPS-induced chemokine concentrations and lower concentrations of all other chemokines/cytokines than control mice [20]. In this study, we demonstrated that LFD feeding reduced the body weight and adiposity of DIO mice; however, there was no significant difference in the expression of the 11 measured cytokines between DIO and DIO + LFD mice, suggesting that DIO mice may be in an early state of obesity. By contrast, significantly dysregulated miRNAs were identified in both groups. Notably, most (23 of 28) of these circulating miRNAs were upregulated in DIO + LFD and downregulated in DIO mice, implying that the downregulation of these miRNAs by obesity could be reversed by
LFD treatment. In addition, target prediction and function annotation revealed that the target genes associated with these 23 differentially expressed miRNAs are involved in metabolic, insulin, and adipocytokine signaling pathways that directly link the pathophysiological changes that occur during obesity and weight reduction. Therefore, whether miRNA supplementation represents a potential therapeutic strategy to treat obesity is an interesting topic requiring further robust investigations to clarify.

As many miRNAs are extensive regulators of adipocyte development and function, their differential expression in the adipose tissue of mice in response to HFD-induced obesity has been explored and discussed in a number of articles [11-13, 21]. For example, in a microarray experiment, 26 miRNAs were upregulated in WAT in response to HFD feeding, including mmu-miR342-3p, mmu-miR-222, mmu-miR-221, mmu-miR-1423p, mmu-miR-142-5p, mmu-miR-21, mmu-miR-335-5p, mmu-miR-146a, mmumiR-146b, mmu-miR-647*, and mmu-miR-379, whereas the following miRNAs were downregulated: mmu-miR-141, mmu-miR-200a, mmumiR-200b, mmumiR-200c, mmu-miR-122, mmu-miR204, mmu-miR-133b, mmu-miR-1, mmu-miR-30a*, mmu-miR-130a, mmu-miR-192, mmu-miR-193a-3p, mmu-miR-203, mmu-miR-378, and mmumiR-30e* [21]. Some of the circulating miRNAs identified in this study have also been reported in the adipose tissue of DIO mice or implicated in adipogenic processes [1113], including Let-7, miR-103, miR-15, the miR-17-92 cluster (miR-17, miR-20a, and miR-92a), miR-21, miR221, and miR-30b. However, these miRNA that were previously reported to be upregulated in adipose tissue were downregulated in DIO mice but upregulated in DIO + LFD mice in this study, casting doubt on the suggestion that these circulating miRNAs originated 


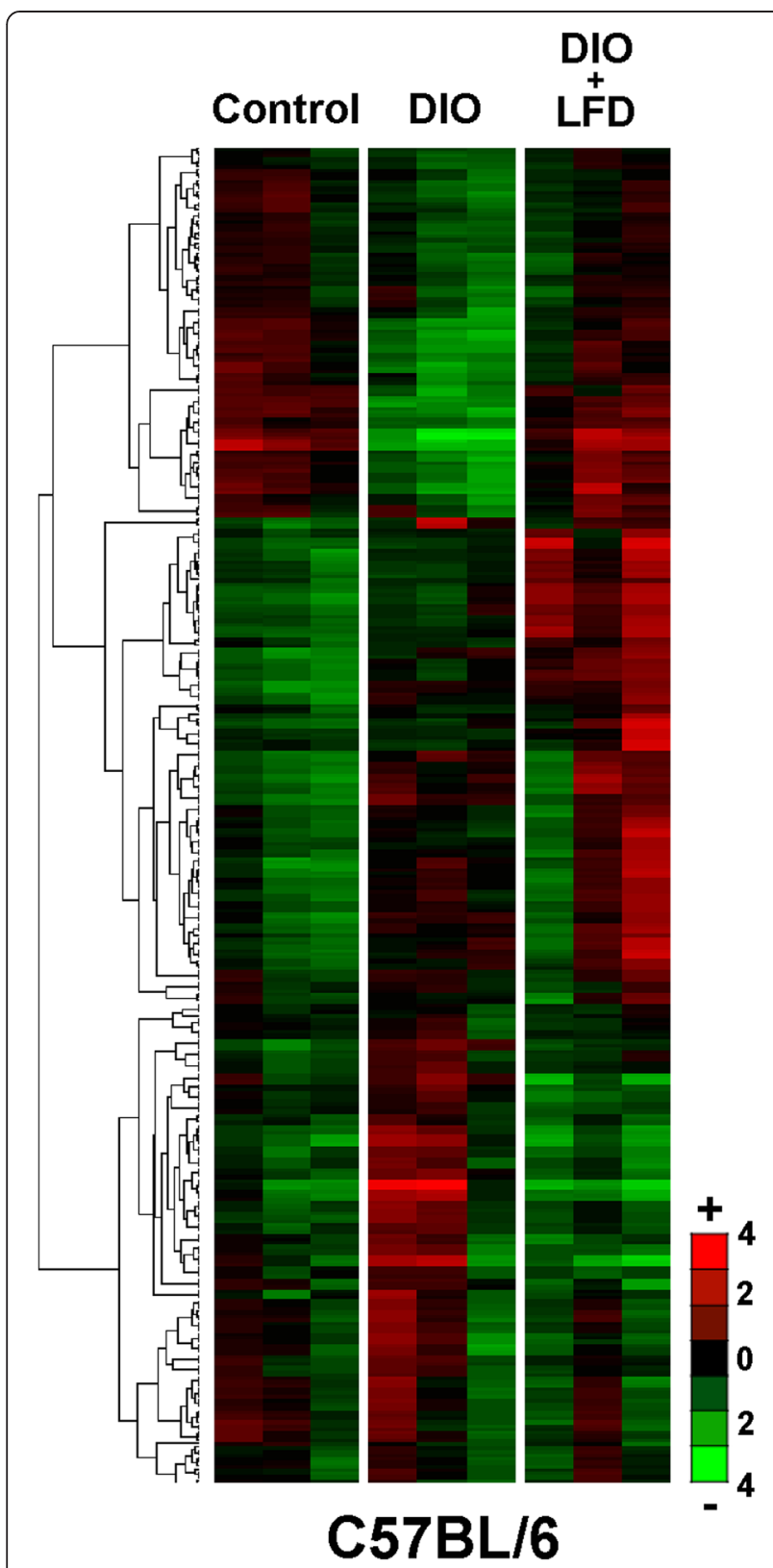

Fig. 6 Hierarchical cluster analysis of differentially expressed circulating miRNAs in the serum of C567BL/6 mice in groups of control, DIO, $\mathrm{DIO}+\mathrm{LFD}(n=3$ in each group)

from the adipose tissue of obese mice. Notably, although the origin of circulating miRNAs is debatable, the expression profile of circulating miRNAs is obviously different from those in pathological tissues [2224]. Whether these circulating miRNAs originated from adipose tissue, blood cells, or other cells of the circulating system requires further experimentation to clarify.

In this study, some of the identified dysregulated miRNAs have been linked to obesity or adipogenesis in the literature. Among them, five members of the Let-7 family (mmu-let-7a, mmu-let-7b, mmu-let-7c, mmu-let-7f,
Table 1 miRNA targets dys-regulated more than 2-fold in serum of C57BL/6NCrl mice at 12w high-fat diet-induced obesity vs. control $(P$-value $<0.05)$

\begin{tabular}{|c|c|c|c|c|c|}
\hline \multicolumn{3}{|l|}{ Up-regulated } & \multicolumn{3}{|l|}{ Down-regulated } \\
\hline $\mathrm{DIO}$ & $\begin{array}{l}\text { Fold } \\
\left(\log _{2}\right) \\
\end{array}$ & $p$-value & $\mathrm{DIO}$ & $\begin{array}{l}\text { Fold } \\
\left(\log _{2}\right) \\
\end{array}$ & $p$-value \\
\hline mmu-miR-711 & 2.15 & 0.00 & mmu-let-7i & -3.98 & 0.01 \\
\hline mmu-miR-712 & 1.58 & 0.04 & mmu-miR-16 & -3.83 & 0.01 \\
\hline mmu-miR-713 & 1.41 & 0.03 & mmu-miR-15a & -3.02 & 0.01 \\
\hline mmu-miR-714 & 1.12 & 0.05 & mmu-miR-26a & -2.61 & 0.00 \\
\hline mmu-miR-715 & 1.07 & 0.01 & mmu-miR-107 & -2.53 & 0.00 \\
\hline mmu-miR-716 & 1.05 & 0.04 & mmu-miR-106b & -2.51 & 0.01 \\
\hline mmu-miR-717 & 1.05 & 0.05 & mmu-miR-17 & -2.49 & 0.00 \\
\hline \multirow[t]{27}{*}{ mmu-miR-574 } & 1.03 & 0.03 & mmu-miR-93 & -2.36 & 0.00 \\
\hline & & & mmu-let-7b & -2.33 & 0.00 \\
\hline & & & mmu-miR-15b & -2.31 & 0.02 \\
\hline & & & mmu-miR-25 & -2.26 & 0.04 \\
\hline & & & mmu-let-7c & -2.20 & 0.00 \\
\hline & & & mmu-miR-20a & -2.17 & 0.00 \\
\hline & & & mmu-miR-103 & -2.16 & 0.00 \\
\hline & & & mmu-miR-221 & -1.97 & 0.00 \\
\hline & & & mmu-miR-195 & -1.86 & 0.03 \\
\hline & & & mmu-miR-21 & -1.85 & 0.00 \\
\hline & & & mmu-miR-106a & -1.82 & 0.00 \\
\hline & & & mmu-let-7a & -1.70 & 0.01 \\
\hline & & & mmu-let-7f & -1.67 & 0.04 \\
\hline & & & mmu-miR-30c & -1.55 & 0.05 \\
\hline & & & mmu-let-7d & -1.52 & 0.02 \\
\hline & & & mmu-miR-19b & -1.41 & 0.01 \\
\hline & & & mmu-miR-30e & -1.35 & 0.02 \\
\hline & & & mmu-miR-30a & -1.34 & 0.02 \\
\hline & & & mmu-miR-23b & -1.32 & 0.03 \\
\hline & & & mmu-miR-486 & -1.28 & 0.03 \\
\hline & & & mmu-miR-30b & -1.25 & 0.01 \\
\hline & & & mmu-miR-130b & -1.25 & 0.05 \\
\hline & & & mmu-miR-106b* & -1.14 & 0.02 \\
\hline & & & mmu-miR-185 & -1.10 & 0.03 \\
\hline & & & mmu-miR-18a & -1.06 & 0.01 \\
\hline & & & mmu-miR-17* & -1.04 & 0.04 \\
\hline & & & mmu-miR-148b & -1.01 & 0.04 \\
\hline
\end{tabular}

and mmu-let-7i) were dysregulated in response to obesity and weight reduction following LFD feeding. Mice with global overexpression of Let-7 are viable, but they have reduced body size and weight [25]. In mice, 12 genes encode members of the Let-7 family, which includes nine slightly different miRNAs (Let-7a, Let-c, and Let-7f [all encoded by two genes], and Let-7b, Let-7d, 
Table 2 miRNA targets dys-regulated more than 2-fold in serum of $\mathrm{C} 57 \mathrm{BL} / 6 \mathrm{NCrl}$ mice following $4 \mathrm{w}$ low-fat diet after 8 w high-fat diet-induced obesity vs. 12 w high-fat diet-induced obesity $(P$-value $<0.05)$

\begin{tabular}{|c|c|c|c|c|c|}
\hline \multicolumn{3}{|l|}{ Up-regulated } & \multicolumn{3}{|l|}{ Down-regulated } \\
\hline $\mathrm{DIO}+\mathrm{LFD}$ & $\begin{array}{l}\text { Fold } \\
\left(\log _{2}\right)\end{array}$ & $\overline{p \text {-value }}$ & $\overline{D I O}+\mathrm{LFD}$ & $\begin{array}{l}\text { Fold } \\
\left(\log _{2}\right)\end{array}$ & $p$-value \\
\hline mmu-miR-16 & 3.48 & 0.00 & mmu-miR-1983 & -3.29 & 0.05 \\
\hline mmu-let-7i & 3.23 & 0.01 & mmu-miR-5112 & -2.39 & 0.02 \\
\hline mmu-miR-26a & 2.57 & 0.02 & mmu-miR-1894 & -2.18 & 0.02 \\
\hline mmu-miR-17 & 2.38 & 0.02 & mmu-miR-5109 & -1.88 & 0.01 \\
\hline mmu-miR-107 & 2.35 & 0.02 & mmu-miR-711 & -1.80 & 0.00 \\
\hline mmu-miR-451 & 2.34 & 0.00 & mmu-miR-351* & -1.37 & 0.04 \\
\hline mmu-miR-195 & 2.28 & 0.01 & mmu-miR-700 & -1.19 & 0.01 \\
\hline mmu-miR-20a & 2.06 & 0.01 & mmu-miR-1940 & -1.15 & 0.03 \\
\hline mmu-miR-25 & 1.88 & 0.03 & mmu-miR-204* & -1.14 & 0.02 \\
\hline mmu-miR-15b & 1.85 & 0.01 & mmu-miR-125b & -1.09 & 0.03 \\
\hline mmu-miR-15a & 1.83 & 0.01 & & & \\
\hline mmu-let-7b & 1.73 & 0.00 & & & \\
\hline mmu-let-7a & 1.69 & 0.00 & & & \\
\hline mmu-let-7c & 1.64 & 0.02 & & & \\
\hline mmu-miR-103 & 1.61 & 0.01 & & & \\
\hline mmu-let-7f & 1.57 & 0.01 & & & \\
\hline mmu-miR-106a & 1.56 & 0.01 & & & \\
\hline mmu-miR-106b & 1.48 & 0.04 & & & \\
\hline mmu-miR-93 & 1.37 & 0.01 & & & \\
\hline mmu-miR-23b & 1.34 & 0.02 & & & \\
\hline mmu-miR-21 & 1.23 & 0.01 & & & \\
\hline mmu-miR-223 & 1.18 & 0.04 & & & \\
\hline mmu-miR-30b & 1.16 & 0.02 & & & \\
\hline mmu-miR-221 & 1.11 & 0.01 & & & \\
\hline mmu-miR-19b & 1.06 & 0.02 & & & \\
\hline mmu-miR-92a & 1.05 & 0.02 & & & \\
\hline mmu-miR-200c & 1.03 & 0.02 & & & \\
\hline mmu-miR-873 & 1.00 & 0.04 & & & \\
\hline
\end{tabular}

Let-7e, Let-7g, Let-7i, and miR-98 [all encoded by one gene]). All Let-7 family members are believed to have similar functions because they share a common seed region (nucleotides 2-8), which mediates interactions between miRNA and target mRNAs [25]. Furthermore, Let-7 transgenic mice exhibit impaired glucose tolerance because of diminished glucose-induced insulin secretion, and anti-miR-induced silencing of Let-7 has been proven to improve blood glucose levels and insulin resistance in obese mice [25].

In vivo, miR-103 is downregulated in the mature adipocytes of obese mice [26] and upregulated during the differentiation of human and murine pre-adipocytes [27, 28].
A 9-fold upregulation of miR-103 was noted during early adipogenesis in 3T3-L1 pre-adipocyte cells, and lipid droplet formation was accelerated when it was ectopically expressed [26]. miR-103 is also upregulated during porcine adipogenesis, and its inhibition suppresses adipogenesis [26].

miR-15a overexpression leads to a decrease in the number, but an increase in the size, of murine adipocytes by inhibiting Delta-like 1 homolog expression [29]. In a study of miRNA libraries reconstructed from preand post-differentiated 3T3-L1 cells, it was noted that miR-15a may not be related to the actual differentiation process, but it may induce growth arrest and/or hormonal stimulation [30]. In addition, the miR-17-19 cluster, which comprises seven miRNAs (miR-17-5p, miR-17-3p, miR-18, miR-19a, miR-20, miR-19b, and miR-92-1) and promotes cell proliferation in various cancers, has been demonstrated to be significantly upregulated at the clonal expansion stage of adipocyte differentiation. MiR17-92 has been revealed to target $\mathrm{Rb} 2 / \mathrm{p} 130$, an important early regulator of pre-adipocyte clonal expansion [31], and the stable transfection of 3T3L1 cells with miR-17-92 resulted in accelerated differentiation and increased triglyceride accumulation after hormonal stimulation [32]. The adipogenic miR-21 has also been demonstrated to be upregulated in human obesity [33] and to enhance adipogenesis in human adipose tissuederived mesenchymal stem cells (hASCs) by mediating TGF- $\beta$ signaling [34].

The miR-30 family has been found to be important for adipogenesis [12]. In this study, miR-30a, miR-30b, and miR-30c were significantly downregulated in obese mice, and miR-30b was significantly upregulated after LFD feeding. MiR-30 family members are strongly upregulated during adipogenesis in human cells, and inhibition of miR-30 inhibits adipogenesis [12]. miR-30 family members have also been demonstrated to act as positive regulators of adipocyte differentiation in a human adipose tissue-derived stem cell model [35]. Overexpression of miR-30a and miR-30d stimulates adipogenesis, and it has been demonstrated that miR-30a and miR-30d target RUNX2, a major regulator of osteogenesis and a potent inhibitor of PPAR $\gamma$, the master gene in adipogenesis [36]. MiR-30c has been found to be upregulated in adipogenesis and to enhance adipogenesis in hASCs, and it appears to target two genes (PAI-1 and ALK2) in distinct pathways [37]. Moreover, miR-30d has been identified as a positive regulator of insulin transcription [38].

Furthermore, in this study, eight miRNAs (mmu-miR-711, mmu-miR-712, mmu-miR-713, mmu-miR-714, mmu-miR715, mmu-miR-716, mmu-miR-717, and mmu-miR-574) were upregulated in DIO mice. Of these, miR-712 is a mechanosensitive miRNA that is upregulated in endothelial cells by disturbed flow, which regulates 


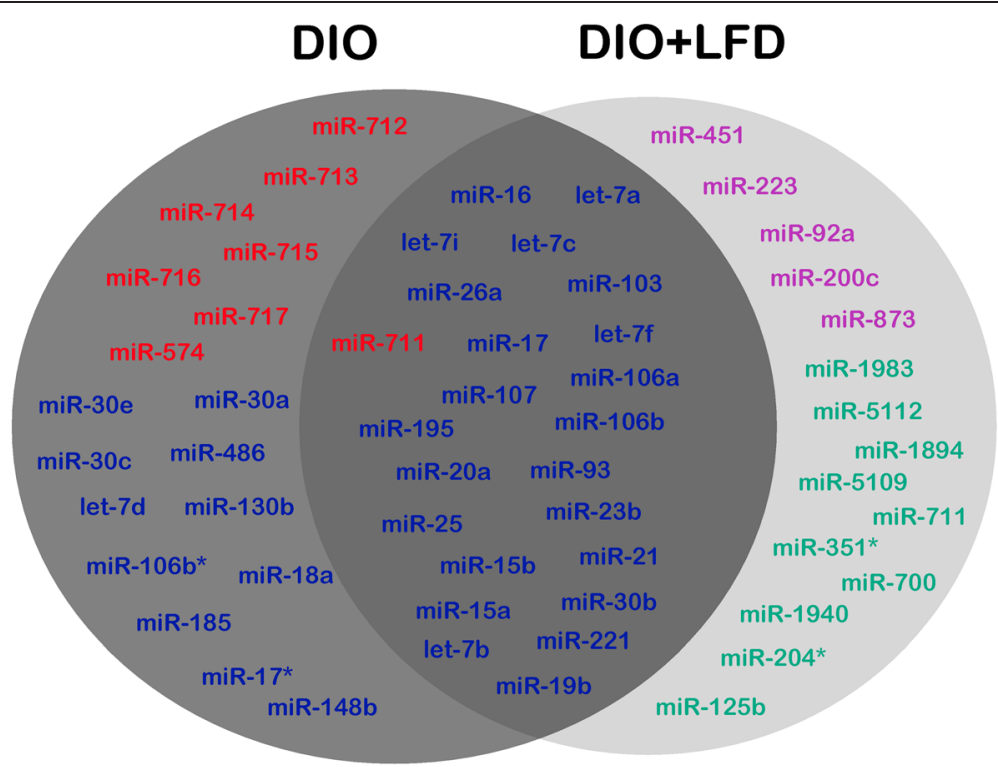

Fig. 7 Venn diagram of the changed miRNAs in the serum of C567BL/6 mice in groups of DIO and DIO + LFD

endothelial dysfunction and atherosclerosis [39, 40]. MiR-717, which was first reported in mice, is encoded by intron 3 of the body mass-associated glypican-3 (Gpc3) gene, and it plays an important regulatory role in renal osmoregulation. Meanwhile, Gpc3 knockout mice display increased body mass, renal dysplasia, and perinatal mortality [41]. Bioinformatics analysis enables functional annotation of MiR-717 orthologs to determine the effect of its target genes on fat-related traits [42]. However, the effects and mechanisms of these eight upregulated miRNAs in the obese mice in this study are poorly understood.

\section{Conclusion}

This study identified the expression profile of circulating miRNAs in a mouse model of DIO and DIO with

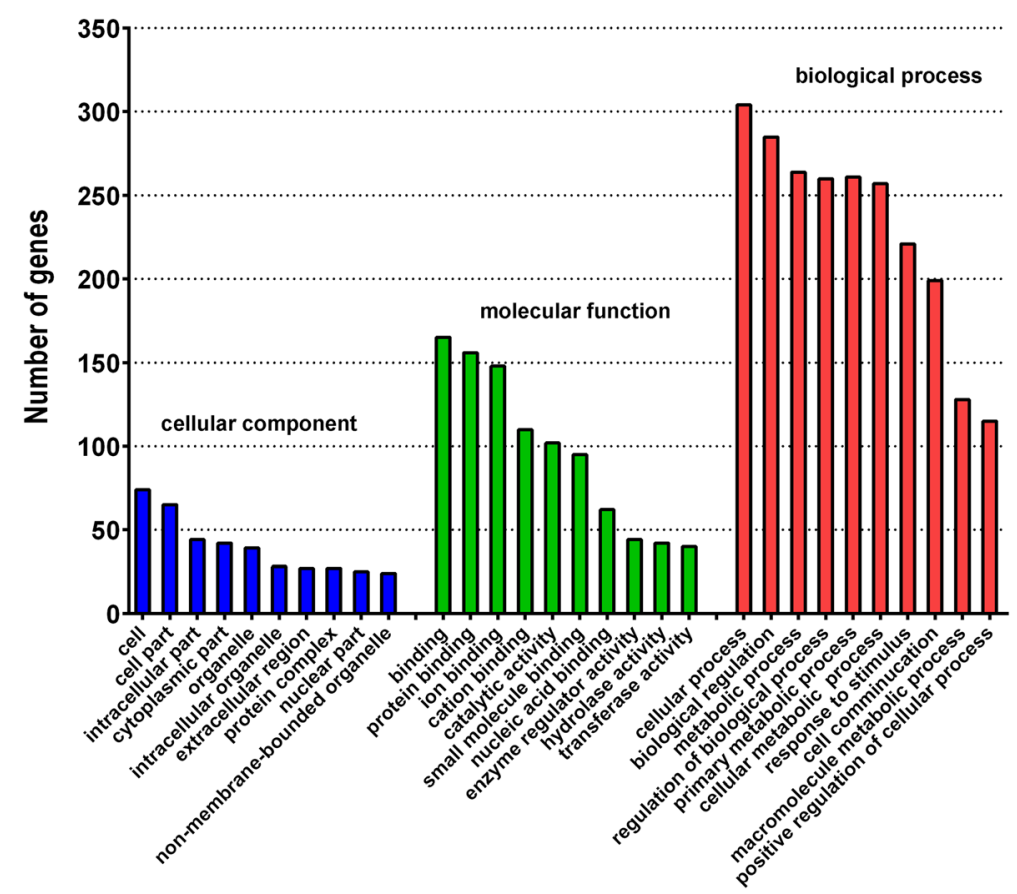

Fig. 8 Partial gene ontology $(\mathrm{GO})$ classification annotated for the predicted target genes of 23 interesting up-regulated miRNAs. The figure shows partial GO enrichment for the predicted target genes in cellular component, molecular function, and biological process. All the $P<0.001$ 
Table 3 The most enriched KEGG pathways of target genes for 23 differentially expressed miRNAs between the sera of C57BL/ $6 \mathrm{NCrl}$ mice during obesity and weight reduction by low-fat diet

\begin{tabular}{|c|c|c|c|}
\hline Pathway & Count of genes & $P$-value & Pathway ID \\
\hline Metabolic pathways & 1024 & $2.11 \mathrm{E} 06$ & ko01100 \\
\hline MAPK signaling pathway & 514 & 1.82E05 & ko04010 \\
\hline Regulation of actin cytoskeleton & 471 & 3.09E05 & ko04810 \\
\hline $\begin{array}{l}\text { Biosynthesis of secondary } \\
\text { metabolites }\end{array}$ & 392 & 4.72E05 & ko05200 \\
\hline Focal adhesion & 342 & $6.21 \mathrm{E} 10$ & ko04510 \\
\hline Insulin signaling pathway & 318 & $5.13 \mathrm{E} 05$ & ko04910 \\
\hline Calcium signaling pathway & 295 & $5.34 \mathrm{E} 05$ & ko04020 \\
\hline $\begin{array}{l}\text { Cytokine-cytokine receptor } \\
\text { interaction }\end{array}$ & 228 & 2.16E04 & ko04060 \\
\hline Tight junction & 172 & 4.22E06 & ko04530 \\
\hline Phagosome & 144 & 2.14E05 & ko04145 \\
\hline $\begin{array}{l}\text { Adipocytokine signaling } \\
\text { pathway }\end{array}$ & 117 & 1.27E04 & ko04920 \\
\hline
\end{tabular}

subsequent weight reduction through LFD feeding. The results demonstrated that the majority of miRNA downregulation in association with obesity could be reversed by LFD feeding. Target prediction and function annotation revealed that the target genes associated with these 23 differentially expressed miRNAs are involved in metabolic, insulin, and adipocytokine signaling pathways that directly link the pathophysiological changes that occur during obesity and weight reduction.

\section{Additional file}

Additional file 1: Correlation of the miRNA expression in the microarray and qPCR. (TIFF $83 \mathrm{~kb}$ ).

\section{Competing interests}

The authors declare that they have no competing interests.

\section{Authors' contributions}

$\mathrm{CHH}$ was responsible for the design and coordination of the data acquisition and analysis, as well as writing and revising the manuscript. CSR contributed to the drafting of the manuscript. SCW was responsible for the animal study and revision of the manuscript. JCY participated by providing and coordinating the resources. CJW and CWL were involved in the acquisition of microRNA array data. THL, YCW, and SLT contributed to the acquisition of the study specimens. All authors read and approved the final manuscript.

\section{Acknowledgements}

The work was supported by a grant from Chang Gung Memorial Hospital (CMRPG8B1421 awarded to Ching-Hua Hsieh and CMRPG8C0351 awarded to Cheng-Shyuan Rau) and a grant from E-Da Hospital (EDPJ104021 awarded to Seng-Feng Jeng).

\section{Author details}

${ }^{1}$ Department of Plastic and Reconstructive Surgery, Kaohsiung Chang Gung Memorial Hospital and Chang Gung University College of Medicine, No. 123, Ta-Pei Road, Niao-Song District, Kaohsiung City 833, Taiwan. ${ }^{2}$ Department of Neurosurgery, Kaohsiung Chang Gung Memorial Hospital and Chang Gung University College of Medicine, No. 123, Ta-Pei Road, Niao-Song District, Kaohsiung City 833, Taiwan. ${ }^{3}$ Department of Anesthesiology, Kaohsiung
Chang Gung Memorial Hospital and Chang Gung University College of Medicine, No. 123, Ta-Pei Road, Niao-Song District, Kaohsiung City 833, Taiwan.

Received: 20 October 2014 Accepted: 7 September 2015

Published online: 16 September 2015

\section{References}

1. Tsukumo DM, Carvalho-Filho MA, Carvalheira JB, Prada PO, Hirabara SM, Schenka AA, et al. Loss-of-function mutation in Toll-like receptor 4 prevents diet-induced obesity and insulin resistance. Diabetes. 2007;56(8):1986-98.

2. Davis JE, Braucher DR, Walker-Daniels J, Spurlock ME. Absence of Tlr2 protects against high-fat diet-induced inflammation and results in greater insulin-stimulated glucose transport in cultured adipocytes. J Nutr Biochem. 2011;22(2):136-41.

3. Kershaw EE, Flier JS. Adipose tissue as an endocrine organ. J Clin Endocrinol Metab. 2004;89(6):2548-56.

4. Despres JP, Lemieux I. Abdominal obesity and metabolic syndrome. Nature. 2006;444(7121):881-7.

5. Wajchenberg BL. Subcutaneous and visceral adipose tissue: their relation to the metabolic syndrome. Endocr Rev. 2000;21(6):697-738.

6. Betanzos-Cabrera G, Estrada-Luna D, Belefant-Miller H, Cancino-Diaz JC. Mice fed with a high fat diet show a decrease in the expression of "toll like receptor (TLR)2 and TLR6 mRNAs in adipose and hepatic tissues. Nutr Hosp. 2012;27(4):1196-203.

7. Surwit RS, Kuhn CM, Cochrane C, McCubbin JA, Feinglos MN. Diet-induced type II diabetes in C57BL/6J mice. Diabetes. 1988;37(9):1163-7.

8. Sartipy P, Loskutoff DJ. Monocyte chemoattractant protein 1 in obesity and insulin resistance. Proc Natl Acad Sci U S A. 2003:100(12):7265-70.

9. Weiss EP, Racette SB, Villareal DT, Fontana L, Steger-May K, Schechtman KB, et al. Improvements in glucose tolerance and insulin action induced by increasing energy expenditure or decreasing energy intake: a randomized controlled trial. Am J Clin Nutr. 2006;84(5):1033-42.

10. Muurling M, Jong MC, Mensink RP, Hornstra G, Dahlmans VE, Pijl H, et al. A low-fat diet has a higher potential than energy restriction to improve highfat diet-induced insulin resistance in mice. Metabolism. 2002:51(6):695-701.

11. Peng Y, Yu S, Li H, Xiang H, Peng J, Jiang S. MicroRNAs: emerging roles in adipogenesis and obesity. Cell Signal. 2014;26(9):1888-96.

12. Hilton C, Neville MJ, Karpe F. MicroRNAs in adipose tissue: their role in adipogenesis and obesity. Int J Obes (2005). 2013;37(3):325-32.

13. Alexander $R$, Lodish $H$, Sun L. MicroRNAs in adipogenesis and as therapeutic targets for obesity. Expert Opin Ther Targets. 2011;15(5):623-36.

14. Hsieh CH, Rau CS, Jeng JC, Chen YC, Lu TH, Wu CJ, et al. Whole blood-derived microRNA signatures in mice exposed to lipopolysaccharides. J Biomed Sci. 2012;19(69):1423-0127.

15. Hsieh CH, Yang JC, Jeng JC, Chen YC, Lu TH, Tzeng SL, et al. Circulating microRNA signatures in mice exposed to lipoteichoic acid. J Biomed Sci. 2013;20(2):1423-0127.

16. Mitchell PS, Parkin RK, Kroh EM, Fritz BR, Wyman SK, Pogosova-Agadjanyan EL, et al. Circulating microRNAs as stable blood-based markers for cancer detection. Proc Natl Acad Sci U S A. 2008;105(30):10513-8.

17. Kosaka N, lguchi H, Yoshioka Y, Takeshita F, Matsuki Y, Ochiya T. Secretory mechanisms and intercellular transfer of microRNAs in living cells. J Biol Chem. 2010;285(23):17442-52.

18. Chen $X$, Liang H, Zhang J, Zen K, Zhang CY. Secreted microRNAs: a new form of intercellular communication. Trends Cell Biol. 2012:22(3):125-32.

19. Hoevenaars FP, Keijer J, Herreman L, Palm I, Hegeman MA, Swarts HJ, et al. Adipose tissue metabolism and inflammation are differently affected by weight loss in obese mice due to either a high-fat diet restriction or change to a low-fat diet. Genes \& nutrition. 2014;9(3):391.

20. Fenton Jl, Nunez NP, Yakar S, Perkins SN, Hord NG, Hursting SD. Diet-induced adiposity alters the serum profile of inflammation in C57BL/6N mice as measured by antibody array. Diabetes Obes Metab. 2009:11(4):343-54.

21. Chartoumpekis DV, Zaravinos A, Ziros PG, Iskrenova RP, Psyrogiannis Al, Kyriazopoulou VE, et al. Differential expression of microRNAs in adipose tissue after long-term high-fat diet-induced obesity in mice. PLoS One. 2012;7(4):e34872

22. Hsieh CH, Rau CS, Jeng JC, Chen YC, Lu TH, Wu CJ, et al. Whole blood-derived microRNA signatures in mice exposed to lipopolysaccharides. J Biomed Sci. 2012;19:69. 
23. Hsieh CH, Yang JC, Jeng JC, Chen YC, Lu TH, Tzeng SL, et al. Circulating microRNA signatures in mice exposed to lipoteichoic acid. J Biomed Sci. 2013;20:2.

24. Rau CS, Yang JC, Wu SC, Chen YC, Lu TH, Lin MW, et al. Profiling circulating microRNA expression in a mouse model of nerve allotransplantation. J Biomed Sci. 2013;20:64.

25. Frost RJ, Olson EN. Control of glucose homeostasis and insulin sensitivity by the Let-7 family of microRNAs. Proc Natl Acad Sci U S A. 2011;108(52):21075-80.

26. Xie H, Lim B, Lodish HF. MicroRNAs Induced During Adipogenesis that Accelerate Fat Cell Development Are Downregulated in Obesity. Diabetes. 2009;58(5):1050-7.

27. Esau C, Kang X, Peralta E, Hanson E, Marcusson EG, Ravichandran LV, et al. MicroRNA-143 regulates adipocyte differentiation. J Biol Chem. 2004;279(50):52361-5.

28. Sun T, Fu M, Bookout AL, Kliewer SA, Mangelsdorf DJ. MicroRNA let-7 regulates 3T3-L1 adipogenesis. Mol Endocrinol (Baltimore, Md). 2009;23(6):925-31.

29. Andersen DC, Jensen $\mathrm{CH}$, Schneider M, Nossent AY, Eskildsen T, Hansen $J$, et al. MicroRNA-15a fine-tunes the level of Delta-like 1 homolog (DLK1) in proliferating 3T3-L1 preadipocytes. Exp Cell Res. 2010;316(10):1681-91.

30. Kajimoto K, Naraba H, Iwai N. MicroRNA and 3T3-L1 pre-adipocyte differentiation. RNA (New York, NY). 2006;12(9):1626-32.

31. Prince AM, May JS, Burton GR, Lyle RE, McGehee Jr RE. Proteasomal degradation of retinoblastoma-related p130 during adipocyte differentiation. Biochem Biophys Res Commun. 2002;290(3):1066-71.

32. Wang Q, Li YC, Wang J, Kong J, Qi Y, Quigg RJ, et al. miR-17-92 cluster accelerates adipocyte differentiation by negatively regulating tumorsuppressor Rb2/p130. Proc Natl Acad Sci U S A. 2008;105(8):2889-94.

33. Keller P, Gburcik V, Petrovic N, Gallagher IJ, Nedergaard J, Cannon B, et al. Gene-chip studies of adipogenesis-regulated microRNAs in mouse primary adipocytes and human obesity. BMC Endocr Disord. 2011;11:7.

34. Kim YJ, Hwang SJ, Bae YC, Jung JS. MiR-21 regulates adipogenic differentiation through the modulation of TGF-beta signaling in mesenchymal stem cells derived from human adipose tissue. Stem Cells (Dayton, Ohio). 2009;27(12):3093-102.

35. Zaragosi LE, Wdziekonski B, Brigand KL, Villageois P, Mari B, Waldmann R, et al. Small RNA sequencing reveals miR-642a-3p as a novel adipocytespecific microRNA and miR-30 as a key regulator of human adipogenesis. Genome Biol. 2011;12(7):R64.

36. Enomoto H, Furuichi T, Zanma A, Yamana K, Yoshida C, Sumitani S, et al. Runx2 deficiency in chondrocytes causes adipogenic changes in vitro. J Cell Sci. 2004;117(Pt 3):417-25.

37. Karbiener M, Neuhold C, Opriessnig P, Prokesch A, Bogner-Strauss JG, Scheideler M. MicroRNA-30c promotes human adipocyte differentiation and co-represses PAI-1 and ALK2. RNA Biol. 2011;8(5):850-60.

38. Tang X, Muniappan L, Tang G, Ozcan S. Identification of glucose-regulated miRNAs from pancreatic \{beta\} cells reveals a role for miR-30d in insulin transcription. RNA (New York, NY). 2009;15(2):287-93.

39. Kumar S, Kim CW, Simmons RD, Jo H. Role of Flow-Sensitive microRNAs in Endothelial Dysfunction and Atherosclerosis: Mechanosensitive Athero-miRs. Arterioscler Thromb Vasc Biol. 2014;34(10):2206-16.

40. Son DJ, Kumar S, Takabe W, Kim CW, Ni CW, Alberts-Grill N, et al. The atypical mechanosensitive microRNA-712 derived from pre-ribosomal RNA induces endothelial inflammation and atherosclerosis. Nat Commun. 2013:4:3000

41. Cano-Gauci DF, Song HH, Yang H, McKerlie C, Choo B, Shi W, et al. Glypican-3deficient mice exhibit developmental overgrowth and some of the abnormalities typical of Simpson-Golabi-Behmel syndrome. J Cell Biol. 1999;146(1):255-64.

42. Kunej T, Skok DJ, Horvat S, Dovc P, Jiang Z. The glypican 3-hosted murine mir717 gene: sequence conservation, seed region polymorphisms and putative targets. Int J Biol Sci. 2010;6(7):769-72.

\section{Submit your next manuscript to BioMed Central and take full advantage of:}

- Convenient online submission

- Thorough peer review

- No space constraints or color figure charges

- Immediate publication on acceptance

- Inclusion in PubMed, CAS, Scopus and Google Scholar

- Research which is freely available for redistribution

Submit your manuscript at www.biomedcentral.com/submit 